\title{
Caracterización y tipificación de explotaciones de dehesa asociadas a cooperativas: un caso de estudio en España
}

\section{Characterization and typification of dehesa farms associated to cooperatives: a case study in Spain}

Francisco Maroto-Molina*

Augusto Gómez-Cabrera ${ }^{a}$

José E. Guerrero-Ginel $^{\mathrm{a}}$

Ana Garrido-Varo ${ }^{a}$

José A. Adame-Siles ${ }^{\mathrm{a}}$

Dolores C. Pérez-Marín ${ }^{\mathrm{a}}$

${ }^{a}$ Universidad de Córdoba. Departamento de Producción Animal. ETSIAM. CN IV, km 396, Córdoba, España.

* Autor de correspondencia: g02mamof@uco.es

\section{- Resumen:}

El objetivo fue caracterizar y tipificar un grupo de explotaciones de dehesa asociadas a una cooperativa de cebo, sacrificio y comercialización de terneros, analizando los sistemas de producción de las distintas tipologías de explotaciones. Se realizaron 114 encuestas, en las que se recolectó información sobre la mano de obra, la base animal, la base territorial y el manejo del ganado vacuno en las explotaciones. Se utilizaron estadísticos descriptivos y análisis multivariantes para definir las relaciones entre las variables y establecer tipologías de explotaciones. En general, las explotaciones disponen de una superficie pequeña (224 ha), predominando la tierra en propiedad (73\%) y la mano de obra familiar (61\%). La mayoría 
de las explotaciones combinan varias especies ganaderas, destacando las asociaciones vacuno-porcino ibérico (53\%) y vacuno-ovino-porcino ibérico (25\%). La intensificación de la producción es habitual, como se refleja en la superficie cultivada anualmente (47\% de la tierra arable) y en la carga ganadera ( 0.73 unidades de ganado mayor/ha). Se observa una gran variabilidad entre explotaciones. Se establecieron cuatro tipologías en función de su tamaño, sus estrategias de diversificación de la producción y sus prácticas de manejo. La tipología más numerosa es la formada por las explotaciones más pequeñas (122 ha). La mayoría de las explotaciones grandes de la zona no participan en la cooperativa, o la han abandonado, porque tienen más facilidad que las pequeñas en seguir una estrategia de venta o cebo de los terneros a título individual.

- Palabras clave: Ganadería extensiva, Diversificación, Intensificación, Cadena de valor.

\section{- Abstract:}

The objective was to characterize and typify a group of dehesa farms associated to a cooperative dedicated to the fattening, slaughtering and trading of beef cattle, by studying the production systems of the different farm typologies. One hundred and fourteen (114) farms were surveyed, collecting data on the characteristics of labor, herd, territorial basis and cattle management. Descriptive statistics and multivariate analyses were used to understand the relationships among variables and to define farm typologies. In general, farms are smallsized (224 ha), land-owned (73\%) and family-run (61\%). Most farms have several livestock species, highlighting beef cattle-Iberian pig (53\%) and beef cattle-sheep-Iberian pig (25\%) associations. Production intensification is widespread, as reflected in the average rate of cultivated area ( $47 \%$ of arable land) and stocking rate ( 0.73 livestock units/ha). There is an important variability among farms. Four (4) farm typologies were stablished varying on farm size, production diversification strategies and management practices. The most numerous typology is that of the smallest farms (122 ha). Large farms are not associated to the cooperative, or have left it, because they have better possibilities for fattening and trading of calves at individual level than small farms.

- Key words: Extensive livestock farming, Diversification, Intensification, Value chain.

Recibido 22/06/2017

Aceptado 20/12/2017 


\section{N Introducción N}

La dehesa es un ecosistema propio de los ambientes mediterráneos de la Península Ibérica, que se caracteriza por presentar un estrato arbóreo ralo de especies esclerófilas, fundamentalmente encinas (Quercus ilex L.) y alcornoques (Quercus suber L.), y un estrato inferior de terófitos, originado en la actividad humana de transformación del bosque esclerófilo original $^{(1)}$. La dehesa tiene un uso múltiple, agrícola, ganadero y forestal, si bien en las dehesas de encinas predomina el uso ganadero ${ }^{(2)}$. La dehesa ha sido reconocida como un sistema de Alto Valor Natural, pues provee de servicios ecosistémicos y favorece la conservación de la biodiversidad $^{(3)}$; la ganadería extensiva juega un papel fundamental en la conservación y manejo de estos sistemas ${ }^{(4)}$ y en la fijación de la población rural ${ }^{(5)}$.

Debido a la diversidad y complejidad de los sistemas de producción ganadera extensiva, numerosos estudios científicos han caracterizado y tipificado las explotaciones de dehesa. Milán et $a l^{(6)}$ encuestaron 130 explotaciones de vacuno de razas autóctonas con un tamaño medio de 125 vacas y 548 ha, diferenciando cuatro tipologías de explotaciones en función del tamaño, la productividad de la mano de obra, el grado de especialización y el grado de intensificación. También se muestreó el $10 \%$ de las explotaciones de vacuno ecológico de las dehesas de Andalucía, encontrando un tamaño medio de 99 vacas y 524 ha $^{(7)}$. García et $a l^{(8)}$ encuestaron 206 explotaciones de dehesa, que agruparon en tres tipologías en base a las características de las fincas y el nivel de intensificación.

Los estudios revisados analizan los sistemas de producción tradicionales, y mayoritarios, en los que los animales se producen en la dehesa, pero se ceban y sacrifican en otras explotaciones, en muchos casos lejos de las zonas de origen ${ }^{(9)}$. En los últimos años han aparecido cooperativas de ganaderos dedicadas al cebo, sacrificio y comercialización en común. Estas cooperativas son habituales en el sector ovino ${ }^{(10)}$, pero en el caso de las ganaderías de vacuno de carne son poco frecuentes. Las cooperativas son importantes para la supervivencia de las explotaciones ${ }^{(11,12)}$, pues permiten a los ganaderos avanzar en la cadena de valor. Sin embargo, las características de los sistemas de producción de las explotaciones que deciden asociarse en estas cooperativas han sido poco estudiadas.

El objetivo del presente trabajo fue caracterizar y tipificar un grupo de explotaciones de dehesa asociadas a una cooperativa de cebo, sacrificio y comercialización de terneros, analizando los sistemas de producción de las distintas tipologías de explotaciones que se asocian en estas cooperativas. 


\section{" Material y métodos N}

\section{- a) Área de estudio}

El área de estudio se localiza en el sur de España y comprende dos comarcas de la provincia de Córdoba: Los Pedroches y Valle del Guadiato. Esta área, caracterizada por la abundancia de dehesas de encinas, comprende unos $6,100 \mathrm{~km}^{2}$ con una densidad de población baja de 14 habitantes $/ \mathrm{km}^{2}$. El clima es mediterráneo con veranos secos y cálidos e inviernos templados, siendo la temperatura media anual de $15^{\circ} \mathrm{C}$. La altitud varía de $500 \mathrm{msnm}$ en la zona noroeste a $800 \mathrm{msnm}$ en el sureste. La precipitación media anual es de $550 \mathrm{~mm}$, llegando a $900 \mathrm{~mm}$ en la zona más alta.

Las explotaciones estudiadas están asociadas a una cooperativa que asume el cebo de los terneros (desde aproximadamente los $250 \mathrm{~kg}$ de peso vivo hasta los $550 \mathrm{~kg}$, en el caso de los machos, y los $450 \mathrm{~kg}$ en el de las hembras), su sacrificio y la comercialización de las canales o piezas. Los ganaderos reciben un pago individualizado por ternero que depende de los ingresos por la venta de su canal, que a su vez depende del peso y la conformación de la misma, y de los costes asociados a su cebo, que son función del tiempo que cada animal ha pasado en el cebadero cooperativo hasta alcanzar el peso de sacrificio.

\section{b) Origen de los datos}

Los datos usados se obtuvieron mediante una encuesta a los ganaderos. Los datos hacen referencia al año 2011, si bien para los parámetros con variabilidad interanual, como el peso de los terneros a la entrada en el cebadero comunitario, se utilizó la media de los años 20092011. El cuestionario se diseñó partiendo de una revisión bibliográfica de estudios de caracterización de explotaciones ${ }^{(6,7,8)}$, e incluye datos cuantitativos y cualitativos sobre los siguientes aspectos: mano de obra; base animal incluyendo las diferentes especies ganaderas

que comparten el territorio; base territorial; y manejo de la alimentación, la reproducción y la sanidad en el caso del vacuno, por ser ésta la producción integrada en el sistema 
cooperativo. Además, se registró la localización geográfica de cada explotación. No se utilizaron datos económicos debido a la ausencia de registros fiables a nivel de explotación individual.

La carga ganadera se calculó mediante la metodología más habitual en los estudios de caracterización de explotaciones de dehesa ${ }^{(13,14)}$, que consiste en estandarizar la carga de cada especie, a unidades de ganado mayor (UGM). Los factores de conversión usados fueron: 1 UGM para vacunos mayores de 24 meses; 0.6 UGM para el resto de vacunos; 1 UGM para equinos; 0.15 UGM para ovinos y caprinos; 0.5 UGM para porcinos reproductores; y 0.3 para porcinos en pastoreo. En las dehesas de encinas es habitual la producción de cerdos ibéricos, que se alimentan de bellotas y pasto durante la fase de acabado o montanera (octubre a marzo). Las cerdas reproductoras, destinadas a la producción de lechones, pueden localizarse en las dehesas o en explotaciones intensivas fuera de las mismas. En el primer caso, las cerdas suelen alojarse en cercados de un tamaño relativamente pequeño, por lo que su presión sobre el sistema pastoral es baja ${ }^{(8)}$. Debido a ello, algunos autores optan por excluir las cerdas reproductoras del cálculo de la carga ganadera. En este estudio se ha optado por calcular dos valores de carga ganadera, incluyendo y excluyendo los reproductores porcinos, con el objetivo de enriquecer la discusión de los resultados.

Se realizaron encuestas a todas las explotaciones asociadas a la cooperativa, recogiéndose 114 encuestas válidas, lo que supone un $80 \%$ del total.

\section{-c) Análisis estadístico •}

Se calcularon los promedios, o frecuencias de las clases, las desviaciones y las correlaciones bivariadas para todas las variables disponibles. A continuación, se realizó un análisis multivariante de los datos, consistente en un análisis factorial seguido de un análisis de conglomerados. Se utilizó el paquete estadístico SPSS v18.

El análisis factorial permite analizar la estructura de las correlaciones existentes entre un gran número de variables, definiendo una nueva serie de variables independientes, conocidas como factores, con una pérdida de información mínima. Debido a la existencia de variables cualitativas, se usó el análisis de correspondencias múltiples (ACM), siendo el porcentaje de varianza explicada, el criterio que define el número de factores a extraer. Las variables cuantitativas se dividieron en tres clases en base a los valores del primer y el tercer cuartil. Los datos de la variable transformada representan las frecuencias de las observaciones por debajo del primer cuartil, entre el primer y el tercer cuartil, y por encima del tercer cuartil, 
tal y como sugieren Solano et $a l^{(15)}$. Las variables binarias se transformaron en variables ordinales, definidas como el número de técnicas de gestión de la alimentación, la reproducción o la sanidad, respectivamente, que usa cada explotación. La selección de las variables incluidas en el ACM se basó en los siguientes criterios: que sean variables discriminativas, es decir, que tengan un coeficiente de variación alto, que no tengan una correlación muy alta con otras variables, y que sean relevantes en cuanto a la descripción del sistema de producción.

A continuación, se realizó un análisis de conglomerados con el fin de agrupar las observaciones con características similares. Se utilizó el método de Ward, que minimiza las diferencias intragrupo, usando la distancia euclídea como medida de similitud. Las variables usadas en el análisis de conglomerados fueron los factores obtenidos en el ACM. El análisis de varianza de un factor (ANOVA) y la prueba de Waller-Duncan se usaron para estudiar la significación de las diferencias entre las medias de las variables cuantitativas para cada grupo. En el caso de las variables cualitativas se usó la prueba Ji-cuadrada.

\section{N Resultados N}

\section{- a) Caracterización general de las explotaciones •}

Los Cuadros 1 y 2 presentan los estadísticos descriptivos de las variables cuantitativas y cualitativas para el conjunto de las explotaciones. Los altos valores de la desviación estándar de algunas variables sugieren la coexistencia de distintas tipologías de explotaciones.

La mayoría de los titulares de explotación son hombres, siendo la forma jurídica más habitual la de persona física. La edad media de los ganaderos es alta, con sólo $11 \%$ de los ganaderos por debajo de los 40 años. La mano de obra es fundamentalmente familiar. Casi el $40 \%$ de los ganaderos obtiene más de la mitad de sus rentas de actividades no relacionadas con la ganadería. De estos, la mitad trabaja en el sector servicios, destacando los comerciales y los veterinarios, y un tercio reciben una pensión de jubilación. La mitad de los ganaderos es usuario de nuevas tecnologías: internet y correo electrónico. Se observa una correlación significativa entre el uso de estas herramientas y la edad del ganadero $(R=-0.29)$. 
Cuadro 1: Estadísticos descriptivos de las variables cuantitativas

\begin{tabular}{|c|c|c|c|c|}
\hline Variables & $\mathrm{N}^{0} 1$ & Media & DE & $\mathrm{ACM}^{2}$ \\
\hline Edad ganadero, años & 112 & 53.4 & 12.5 & \\
\hline $\mathrm{N}^{0}$ vacas & 114 & 55.4 & 57.5 & * \\
\hline $\mathrm{N}^{0}$ vacas raza $3 / \mathrm{N}^{0}$ vacas, $\%$ & 114 & 26.4 & 40.6 & * \\
\hline$N^{0}$ toros & 114 & 2.6 & 2.6 & \\
\hline $\mathrm{N}^{0}$ toros raza $3 / \mathrm{N}^{0}$ toros, $\%$ & 114 & 98.7 & 10.4 & \\
\hline $\mathrm{N}^{0}$ novillas & 114 & 10.2 & 10.3 & \\
\hline $\mathrm{N}^{0}$ novillas raza $3 / \mathrm{N}^{0}$ novillas, $\%$ & 114 & 35.5 & 47.7 & \\
\hline $\mathrm{N}^{0}$ derechos nodriza ${ }^{4}$ & 114 & 40.5 & 42.6 & \\
\hline $\mathrm{N}^{0}$ derechos nodriza $4 / \mathrm{N}^{0}$ hembras, $\%$ & 114 & 62.7 & 30.5 & * \\
\hline $\mathrm{N}^{0}$ vacas por toro & 114 & 22.4 & 10.7 & * \\
\hline $\mathrm{N}^{0}$ novillas por vaca & 114 & 0.22 & 0.19 & * \\
\hline$N^{0}$ ovejas & 32 & 257.1 & 280.0 & * \\
\hline $\mathrm{N}^{0}$ cabras & 3 & 120.0 & 113.6 & \\
\hline $\mathrm{N}^{0}$ cerdas reproductoras & 58 & 119.4 & 109.8 & * \\
\hline $\mathrm{N}^{0}$ cerdos montanera & 46 & 204.7 & 376.2 & * \\
\hline$N^{0}$ equinos & 7 & 25.6 & 17.1 & \\
\hline UGM totales & 114 & 132.1 & 152.2 & \\
\hline UGM vacuno/UGM totales, $\%$ & 114 & 57.2 & 25.1 & * \\
\hline UGM ovino/UGM totales, \% & 114 & 7.2 & 15.0 & * \\
\hline UGM porcino/UGM totales, $\%$ & 114 & 33.9 & 23.5 & \\
\hline Superficie pastos naturales en propiedad, ha & 114 & 97.3 & 274.9 & \\
\hline Superficie pastos naturales arrendada, ha & 114 & 24.9 & 61.0 & \\
\hline Superficie cultivo en propiedad, ha & 114 & 67.0 & 108.9 & \\
\hline Superficie cultivo arrendada, ha & 114 & 35.0 & 103.2 & \\
\hline Superficie cultivada cada año, ha & 60 & 33.1 & 33.6 & \\
\hline Superficie total, ha & 114 & 224.1 & 313.4 & * \\
\hline Superficie pastos naturales/superficie total, $\%$ & 114 & 55.1 & 42.2 & \\
\hline Superficie cultivo/superficie total, $\%$ & 114 & 44.9 & 42.2 & * \\
\hline Superficie en propiedad/superficie total, $\%$ & 114 & 72.8 & 36.8 & \\
\hline Superficie arrendada/superficie total, $\%$ & 114 & 27.2 & 36.8 & * \\
\hline Superficie cultivada cada año/superficie cultivo, \% & 60 & 46.7 & 37.2 & \\
\hline UGM/superficie total (sin cerdas reproductoras) & 114 & 0.52 & 0.21 & * \\
\hline UGM/superficie total (con cerdas reproductoras) & 114 & 0.73 & 0.39 & * \\
\hline $\mathrm{N}^{0}$ cerdos montanera/superficie total & 46 & 0.80 & 0.43 & \\
\hline Duración cubrición vacas, meses & 37 & 6.6 & 1.2 & \\
\hline $\mathrm{N}^{0}$ terneros destetados por año & 97 & 43.0 & 38.0 & \\
\hline $\mathrm{N}^{0}$ terneros destetados por vaca & 97 & 0.83 & 0.17 & \\
\hline $\mathrm{N}^{0}$ vacas desechadas por año & 91 & 4.3 & 3.4 & \\
\hline $\mathrm{N}^{0}$ vacas desechadas $/ \mathrm{N}^{0}$ vacas, $\%$ & 91 & 9.3 & 4.7 & \\
\hline Peso terneros entrada cebadero, $\mathrm{kg}$ & 99 & 260.1 & 36.1 & \\
\hline
\end{tabular}

1 La media y la desviación estándar (DE) se refieren sólo a aquellas explotaciones que disponen de un dato distinto de cero para la variable en cuestión.

2 Variables incluidas en el análisis de correspondencias múltiples.

${ }^{3}$ Animales de razas foráneas especializadas, Charolais o Limousin.

${ }^{4}$ Derechos de pago a vacas nodrizas de la Política Agraria Común(16).

UGM= unidades de ganado mayor. 
Cuadro 2: Porcentaje de explotaciones en cada clase de las variables cualitativas

\begin{tabular}{|c|c|c|c|c|}
\hline Variables & $\mathrm{N}^{0}$ datos $^{1}$ & Clases & $\%$ & $\mathrm{ACM}^{2}$ \\
\hline \multirow[t]{2}{*}{ Sexo ganadero } & 82 & Hombre & 68 & \\
\hline & & Mujer & 32 & \\
\hline \multirow{4}{*}{ Forma jurídica } & 114 & Persona física & 72 & \\
\hline & & Sociedad civil privada & 14 & \\
\hline & & Comunidad de bienes & 8 & \\
\hline & & Sociedad limitada & 6 & \\
\hline Agricultor título principal ${ }^{3}$ & 114 & SíNo ${ }^{4}$ & 61 & \\
\hline \multirow{4}{*}{ Otras actividades } & 45 & Agricultura & 14 & \\
\hline & & Industria & 0 & \\
\hline & & Servicios & 50 & \\
\hline & & Jubilado & 36 & \\
\hline \multirow{3}{*}{ Tipo mano de obra } & 114 & Familiar & 61 & \\
\hline & & Mixta & 26 & \\
\hline & & Contratada & 13 & \\
\hline Usuario TIC & 114 & SilNo ${ }^{4}$ & 51 & \\
\hline \multirow{3}{*}{ Evolución prevista rebaño } & 114 & Aumentar & 22 & * \\
\hline & & Mantener & 68 & \\
\hline & & Decrecer & 10 & \\
\hline Presencia ovino & 114 & $\mathrm{Si} / \mathrm{No}^{4}$ & 28 & \\
\hline Presencia caprino & 114 & $\mathrm{Si} / \mathrm{No}^{4}$ & 3 & \\
\hline Presencia porcino reproductor & 114 & $\mathrm{Si} / \mathrm{No}^{4}$ & 51 & \\
\hline Presencia porcino montanera & 114 & SíNo ${ }^{4}$ & 40 & \\
\hline Presencia equinos & 114 & $\mathrm{Si} / \mathrm{No}^{4}$ & 6 & \\
\hline Monta dirigida ${ }^{5}$ & 71 & $\mathrm{Si} / \mathrm{No}^{4}$ & 30 & \\
\hline Cubrición estacional & 114 & $\mathrm{Si} / \mathrm{No}^{4}$ & 32 & * \\
\hline Diagnóstico gestación & 114 & Sí/No ${ }^{4}$ & 14 & * \\
\hline Aporte concentrados antes destete & 114 & SíNo ${ }^{4}$ & 58 & * \\
\hline Aporte concentrados después destete & 114 & Sí/No ${ }^{4}$ & 75 & * \\
\hline Oficialmente indemne tuberculosis ${ }^{6}$ & 114 & SíNo ${ }^{4}$ & 91 & * \\
\hline Oficialmente indemne brucelosis 6 & 114 & $\mathrm{Sí} / \mathrm{No}^{4}$ & 94 & * \\
\hline Plan vacunación vacas & 114 & SíNo ${ }^{4}$ & 83 & * \\
\hline Plan vacunación terneros & 114 & $\mathrm{Si} / \mathrm{No}^{4}$ & 46 & * \\
\hline Plan desparasitación vacas & 114 & $\mathrm{Si} / \mathrm{No}^{4}$ & 75 & * \\
\hline Plan desparasitación terneros & 114 & $\mathrm{Si} / \mathrm{NO}^{4}$ & 52 & * \\
\hline
\end{tabular}

${ }^{1}$ El porcentaje se refiere sólo a aquellas explotaciones que disponen de un dato distinto de cero para la variable en cuestión.

2 Variables incluidas en el análisis de correspondencias múltiples.

${ }^{3}$ Ganaderos con más del $50 \%$ de las rentas procedentes de la actividad agraria.

${ }^{4}$ En las variables dicotómicas el porcentaje se refiere a las respuestas afirmativas.

${ }^{5}$ Control de la paternidad utilizando un único toro para cada lote de vacas.

${ }^{6}$ Calificación sanitaria oficial de acuerdo a la legislación española(17).

$\mathrm{TIC}=$ usuarios de las tecnologías de la información y la comunicación. 
La superficie total media de las explotaciones es de 224 ha, aunque se observa una gran variabilidad. La tasa de superficie en propiedad es alta, pues el $57 \%$ de los ganaderos no arriendan tierras y sólo en el $25 \%$ de los casos la superficie arrendada es superior al $50 \%$ del total. En el $29 \%$ de las explotaciones toda la superficie corresponde a pastos naturales, en los que el ganado aprovecha la vegetación espontánea. En el $25 \%$ toda la superficie se cultiva con especies forrajeras mediante un sistema de parcelas rotacionales. El resto de las explotaciones combinan superficie de pastos naturales y superficie de cultivo. Se observa que las dehesas con un bajo porcentaje de superficie de cultivo $(<20 \%)$ se encuentran mayoritariamente en la zona noroccidental de las comarcas estudiadas, donde, además de una menor precipitación anual, se encuentran los suelos más pobres.

El $84 \%$ de los ganaderos explota varias especies conjuntamente, destacando las asociaciones vacuno-porcino ibérico, y vacuno-ovino-porcino ibérico; si bien en algún caso existen otras especies como las cabras y los caballos. Los hatos de vacuno tienen un tamaño medio de 55 vacas y los de ovino de 257 ovejas. En el caso del porcino ibérico, las explotaciones que sólo cuentan con reproductores, el $37 \%$ del total, tienen un censo medio de 139 cerdas; las que sólo producen cerdos de montanera, el $26 \%$, tienen una media de 182 cerdos; y las que cuentan con ambos tipos de animal, tienen en promedio 64 cerdas reproductoras y 247 cerdos de montanera. La carga ganadera es alta, con un promedio de $0.52 \mathrm{UGM} / \mathrm{ha}$ o $0.73 \mathrm{UGM} / \mathrm{ha}$ si se consideran las cerdas reproductoras, y presenta una correlación significativa con la superficie total $(\mathrm{R}=-0.24)$.

La alimentación del ganado se basa en el pastoreo libre y el aporte de suplementos en épocas desfavorables, normalmente en la época estival. Los forrajes cultivados, se consumen en fresco o bien conservados en forma de heno. En conjunto, el $45 \%$ de la superficie de las explotaciones encuestadas es cultivable, de la cual el $47 \%$ se rotura y siembra cada año. Se observa una correlación significativa entre el porcentaje de tierra cultivable de la explotación y el porcentaje de las UGM correspondiente al ovino $(\mathrm{R}=-0.35)$. Como se ha explicado, las dehesas con menos superficie de cultivo se encuentran en la zona de suelos más pobres, donde la vegetación natural suele alcanzar un porte bajo, siendo más adecuada para el ovino que para el vacuno. En el caso del vacuno, la mayor parte de los ganaderos aporta concentrados a los terneros durante o tras la lactación para prepararlos para la fase de cebo intensivo. Los terneros se envían al cebadero comunitario con un peso medio de $260 \mathrm{~kg}$.

Las técnicas de manejo reproductivo del ganado vacuno más frecuentes son la monta libre y la cubrición continua. No obstante, casi un tercio de las explotaciones separan los toros y las vacas durante unos meses al año para evitar partos de finales de primavera y verano. Se observa una correlación significativa entre el uso de la cubrición estacional y el número de vacas $(\mathrm{R}=0.28)$.

En cuanto a la base genética, el $65 \%$ de las vacas procede de cruces de razas autóctonas con toros Charolais o Limousin, mientras que la práctica totalidad de los toros son de pura raza 
(Cuadro 3). Cabe destacar que sólo el $21 \%$ de las vacas descritas como de pura raza por los ganaderos entrevistados están inscritas en el correspondiente Libro Genealógico, mientras que en el caso de los toros este valor es del $94 \%$. En el área de estudio, los ganaderos suelen producir sus propias novillas de reposición, pero los toros son comprados a otras explotaciones para evitar problemas de consanguinidad. Dado que la mayoría de las explotaciones sólo tienen toros Charolais o Limousin, los ganaderos suelen considerar que las novillas son de pura raza después de uno o varios cruces de absorción. El número de novillas presentes por vaca es 0.22 , lo que corresponde a una tasa de reposición en torno al $15 \%$, entendiendo como novillas las hembras de reposición entre 6 y 24 meses de edad. El número medio de vacas por toro es 22 , aunque este dato es muy variable. Se observa una correlación significativa entre el número de vacas y el número de vacas por toro $(\mathrm{R}=0.23)$, ya que incluso en los hatos con pocas nodrizas suele haber al menos un toro, pues no se utiliza la inseminación artificial. El uso de otras técnicas de control reproductivo, como el diagnóstico de gestación, es también minoritario. En promedio, los ganaderos declaran destetar 0.83 terneros por vaca y año. Se observa una correlación significativa de este índice con el porcentaje de vacas de razas autóctonas en el rebaño $(\mathrm{R}=0.27)$. Esta correlación no indica necesariamente que las vacas autóctonas tengan una mejor productividad, pues multitud de factores pueden estar influyendo. Por ejemplo, los ganaderos de la zona tienden a percibir a las vacas Charolais o Limousin como más valiosas que las autóctonas, siendo más reacios a eliminarlas cuando detectan problemas reproductivos.

Cuadro 3: Composición racial de los rebaños

\begin{tabular}{lcccc}
\hline \multirow{2}{*}{ Razas y cruces } & \multicolumn{2}{c}{ \% Animales } & \multicolumn{2}{c}{ \% Explotaciones $^{1}$} \\
& Vacas & Toros & Vacas & Toros \\
\hline Rubia de Aquitania & 0.0 & 1.7 & 0.0 & 0.9 \\
Charolais & 12.8 & 45.3 & 18.4 & 57.9 \\
Limousin & 14.2 & 47.3 & 20.2 & 68.4 \\
Negra andaluza & 1.8 & 1.0 & 0.9 & 0.9 \\
Retinta & 4.9 & 3.7 & 9.6 & 7.0 \\
Simmental & 0.0 & 0.3 & 0.0 & 0.9 \\
Braunvieh x Charolais & 1.1 & 0.0 & 1.8 & 0.0 \\
Braunvieh x Limousin & 0.9 & 0.0 & 0.9 & 0.0 \\
Charolais x Limousin & 18.5 & 0.0 & 14.0 & 0.0 \\
Holstein x Charolais & 0.2 & 0.0 & 0.9 & 0.0 \\
Pajuna x Braunvieh & 0.7 & 0.0 & 0.9 & 0.0 \\
Retinta x Charolais & 3.5 & 0.0 & 5.3 & 0.0 \\
Retinta x Limousin & 5.7 & 0.0 & 6.1 & 0.0 \\
Retinta x Braunvieh & 0.2 & 0.0 & 0.9 & 0.0 \\
Desconocido x Charolais o Limousin & 35.6 & 0.7 & 49.1 & 1.8 \\
\hline
\end{tabular}

1 Los porcentajes no suman 100 porque algunas explotaciones usan varias razas 0 cruces. 
En el aspecto sanitario, casi todas las explotaciones disponen de la máxima calificación sanitaria respecto a brucelosis y tuberculosis ${ }^{(17)}$. El $75 \%$ de las mismas dispone de planes de vacunación y desparasitación de las nodrizas, no así de los terneros. Las enfermedades más frecuentes son la diarrea viral bovina y la parainfluenza en adultos, y la diarrea neonatal en terneros, si bien su prevalencia es baja.

\section{-b) Análisis de correspondencias múltiples •}

Se obtuvieron cinco factores que explican el $69.91 \%$ de la varianza. El primer factor (F1) explica el $17.13 \%$ de la varianza y representa el tamaño de la explotación; las variables que más contribuyen en este primer eje son superficie total, $\mathrm{n}^{\circ}$ vacas, $\mathrm{n}^{\circ}$ cerdos en montanera $\mathrm{y}$ $\mathrm{n}^{\circ}$ cerdas reproductoras. También son importantes las variables relativas al uso de técnicas de gestión reproductiva y a la carga ganadera.

El segundo factor (F2) explica el $17.06 \%$ de la varianza y se refiere a la importancia relativa del ovino. Las variables más importantes en este caso son $n^{\circ}$ ovejas, UGM ovino/UGM totales, UGM vacuno/UGM totales y superficie arrendada/superficie total.

El tercer factor (F3) explica el $12.96 \%$ de la varianza y se corresponde con la importancia relativa del porcino reproductor en las explotaciones. El $\mathrm{n}^{\mathrm{o}}$ cerdas reproductoras y la carga ganadera son las variables con una mayor contribución en este eje. Les siguen las variables UGM vacuno/UGM totales y superficie cultivo/superficie total.

El cuarto factor (F4) explica el $11.74 \%$ de la varianza y representa la presión sobre el sistema pastoral, siendo la variable más importante la carga ganadera excluyendo las cerdas reproductoras. Destaca también en este eje la variable $n^{\circ}$ derechos nodriza $/ n^{\circ}$ hembras.

El quinto factor (F5) explica el $11.02 \%$ de la varianza y representa el nivel de intensificación. Las variables con mayor peso son las relativas al uso de técnicas de gestión reproductiva y al manejo sanitario, así como el porcentaje de vacas de razas especializadas en el rebaño y el porcentaje de superficie de cultivo.

\section{-c) Tipologías de explotaciones •}

El análisis de conglomerados jerárquicos permite establecer cuatro tipologías de explotaciones. Los valores medios de las variables cuantitativas para cada tipología se 
presentan en el Cuadro 4 y el porcentaje de explotaciones en cada clase de las variables cualitativas en el Cuadro 5.

Cuadro 4: Promedio de las variables cuantitativas para cada tipología

\begin{tabular}{|c|c|c|c|c|c|}
\hline \multirow[b]{2}{*}{ Variables } & \multicolumn{4}{|c|}{ Grupos } & \multirow[b]{2}{*}{$P$ valor } \\
\hline & 1 & 2 & 3 & 4 & \\
\hline Edad ganadero, años & 54.9 & 53.4 & 51.7 & 55.4 & 0.83 \\
\hline $\mathrm{N}^{0}$ vacas & $40.1^{a b}$ & $64.2^{b}$ & $31.4^{\mathrm{a}}$ & $124.3^{c}$ & 0.00 \\
\hline $\mathrm{N}^{0}$ vacas raza $1 / \mathrm{N}^{0}$ vacas, $\%$ & 25.1 & 31.9 & 16.7 & 31.0 & 0.78 \\
\hline $\mathrm{N}^{0}$ toros & $2.0^{a b}$ & $2.8^{\mathrm{b}}$ & $1.4^{\mathrm{a}}$ & $5.5^{\mathrm{c}}$ & 0.00 \\
\hline $\mathrm{N}^{0}$ toros raza $1 / \mathrm{N}^{0}$ toros, $\%$ & 98.6 & 100.0 & 95.8 & 100.0 & 0.76 \\
\hline $\mathrm{N}^{0}$ novillas & $8.1 \mathrm{ab}$ & $15.0 \mathrm{bc}$ & $3.4^{a}$ & $16.6^{c}$ & 0.00 \\
\hline $\mathrm{N}^{0}$ novillas raza $1 / \mathrm{N}^{0}$ novillas, $\%$ & 30.4 & 52.9 & 27.8 & 52.3 & 0.23 \\
\hline $\mathrm{N}^{0}$ derechos nodriza ${ }^{2}$ & $29.6^{a b}$ & $49.4^{\mathrm{bc}}$ & $20.5^{\mathrm{a}}$ & $66.9 c$ & 0.00 \\
\hline $\mathrm{N}^{0}$ derechos nodriza $2 / \mathrm{N}^{0}$ hembras, $\%$ & 64.2 & 54.1 & 56.7 & 54.1 & 0.41 \\
\hline $\mathrm{N}^{0}$ vacas por toro & 21.9 & 24.6 & 22.5 & 22.0 & 0.82 \\
\hline$N^{0}$ novillas por vaca & 0.22 & 0.26 & 0.14 & 0.14 & 0.24 \\
\hline $\mathrm{N}^{0}$ ovejas & $98.8^{a}$ & $213.3^{\mathrm{ab}}$ & $280.0^{\mathrm{b}}$ & $170.0^{\mathrm{ab}}$ & 0.00 \\
\hline $\mathrm{N}^{0}$ cabras & 40.0 & - & 160.0 & - & 0.58 \\
\hline $\mathrm{N}^{0}$ cerdas reproductoras & $61.5^{\mathrm{a}}$ & $252.1^{\mathrm{b}}$ & $40.6 \mathrm{a}$ & $76.0^{a}$ & 0.00 \\
\hline $\mathrm{N}^{0}$ cerdos montanera & $89.5^{\mathrm{a}}$ & $35.0^{a}$ & $137.5^{\mathrm{ab}}$ & $203.6^{b}$ & 0.00 \\
\hline$N^{0}$ equinos & 16.8 & 31.0 & - & - & 0.31 \\
\hline UGM totales & $74.9 \mathrm{a}$ & $212.0^{\mathrm{b}}$ & $99.6^{a}$ & $205.9 \mathrm{~b}$ & 0.00 \\
\hline UGM vacuno/UGM totales, $\%$ & $67.6^{\mathrm{b}}$ & $34.6^{a}$ & $35.6^{\mathrm{a}}$ & $66.9 \mathrm{~b}$ & 0.00 \\
\hline UGM ovino/UGM totales, $\%$ & $2.3^{a}$ & $2.9 \mathrm{a}$ & $37.2^{\mathrm{b}}$ & $2.0^{a}$ & 0.00 \\
\hline UGM porcino/UGM totales, $\%$ & 28.9a & $60.6^{\mathrm{b}}$ & $21.8^{\mathrm{a}}$ & $31.1^{\mathrm{a}}$ & 0.00 \\
\hline Superficie pastos naturales en propiedad, ha & $50.2^{\mathrm{a}}$ & $91.1 \mathrm{ab}$ & $25.2^{\mathrm{a}}$ & $144.8^{b}$ & 0.01 \\
\hline Superficie pastos naturales arrendada, ha & $12.7^{a}$ & $23.6^{a}$ & $74.0^{\mathrm{b}}$ & $33.8 \mathrm{ab}$ & 0.00 \\
\hline Superficie cultivo en propiedad, ha & $46.6^{\mathrm{a}}$ & $82.5^{\mathrm{a}}$ & $25.4^{\mathrm{a}}$ & $182.0^{\mathrm{b}}$ & 0.00 \\
\hline Superficie cultivo arrendada, ha & $12.5^{\mathrm{a}}$ & $57.8^{\mathrm{a}}$ & $49.3^{\mathrm{a}}$ & $64.6^{\mathrm{a}}$ & 0.01 \\
\hline Superficie cultivada cada año, ha & $23.4^{\mathrm{a}}$ & $31.8^{\mathrm{a}}$ & $17.0^{\mathrm{a}}$ & $75.5^{\mathrm{b}}$ & 0.00 \\
\hline Superficie total, ha & $122.0^{a}$ & $255.0^{\mathrm{b}}$ & $173.8^{\mathrm{ab}}$ & $425.1^{c}$ & 0.00 \\
\hline Superficie pastos naturales/superficie total, $\%$ & 50.7 & 61.0 & 65.9 & 47.7 & 0.59 \\
\hline Superficie cultivo/superficie total, $\%$ & 49.3 & 39.0 & 34.1 & 52.3 & 0.59 \\
\hline Superficie en propiedad/superficie total, $\%$ & $81.1^{\mathrm{b}}$ & $66.1^{\mathrm{b}}$ & $27.8^{\mathrm{a}}$ & $76.4^{\mathrm{b}}$ & 0.00 \\
\hline Superficie arrendada/superficie total, \% & 18.9a & $33.9 \mathrm{a}$ & $72.2^{b}$ & $23.6^{\mathrm{a}}$ & 0.00 \\
\hline Superficie cultivada cada año/superficie cultivo, \% & 46.7 & 55.5 & 30.6 & 34.7 & 0.57 \\
\hline UGM/Superficie total (sin cerdas reproductoras) & $0.55^{\mathrm{a}}$ & $0.38^{a}$ & $0.55^{\mathrm{a}}$ & $0.49 \mathrm{a}$ & 0.02 \\
\hline UGM/Superficie total (con cerdas reproductoras) & $0.71 \mathrm{ab}$ & $1.05^{b}$ & $0.63 \mathrm{a}$ & $0.51^{\mathrm{a}}$ & 0.00 \\
\hline $\mathrm{N}^{0}$ cerdos montanera/superficie total & $0.82^{\mathrm{a}}$ & $0.12^{\mathrm{a}}$ & $0.78^{a}$ & $0.53^{a}$ & 0.05 \\
\hline Duración cubrición vacas, meses & 6.5 & 6.2 & - & 7.3 & 0.41 \\
\hline $\mathrm{N}^{0}$ terneros destetados por año & $32.2^{\mathrm{a}}$ & $61.6^{\mathrm{b}}$ & $20.3^{\mathrm{a}}$ & $113.3^{c}$ & 0.00 \\
\hline $\mathrm{N}^{0}$ terneros destetados por vaca & $0.84^{\mathrm{b}}$ & $0.81^{b}$ & $0.74^{a}$ & $0.94 c$ & 0.06 \\
\hline $\mathrm{N}^{0}$ vacas desechadas por año & $3.5^{\mathrm{ab}}$ & $5.3^{b}$ & $1.9^{a}$ & $11.4^{c}$ & 0.00 \\
\hline $\mathrm{N}^{0}$ vacas desechadas $/ \mathrm{N}^{0}$ vacas, $\%$ & 9.9 & 8.3 & 8.9 & 11.0 & 0.63 \\
\hline Peso terneros entrada cebadero, $\mathrm{kg}$ & 258.6 & 264.0 & 264.9 & 260.1 & 0.93 \\
\hline
\end{tabular}

${ }^{1}$ Animales de razas foráneas especializadas, Charolais o Limousin.

${ }^{2}$ Derechos de pago a vacas nodrizas de la Política Agraria Común ${ }^{(16)}$.

$\mathrm{UGM}=$ unidades de ganado mayor.

a,b,c Letras distintas en la misma fila señalan diferencias significativas $(P<0.05)$. 
Cuadro 5: Porcentaje de explotaciones en cada clase de las variables cualitativas para cada tipología

\begin{tabular}{|c|c|c|c|c|c|c|}
\hline \multirow[b]{2}{*}{ Variables } & \multirow[b]{2}{*}{ Clases } & \multicolumn{4}{|c|}{ Grupos } & \multirow[b]{2}{*}{$P$ valor } \\
\hline & & 1 & 2 & 3 & 4 & \\
\hline \multirow[t]{2}{*}{ Sexo ganadero } & Hombre & 67 & 78 & 87 & 60 & \multirow[t]{2}{*}{0.60} \\
\hline & Mujer & 33 & 22 & 13 & 40 & \\
\hline \multirow[t]{4}{*}{ Forma jurídica } & Persona física & 80 & 53 & 66 & 63 & \multirow[t]{4}{*}{0.19} \\
\hline & Sociedad civil privada & 9 & 29 & 17 & 5 & \\
\hline & Comunidad de bienes & 9 & 12 & 0 & 0 & \\
\hline & Sociedad limitada & 2 & 6 & 17 & 12 & \\
\hline Agricultor título principal ${ }^{1}$ & Sí/No2 & 55 & 65 & 92 & 63 & 0.12 \\
\hline \multirow{4}{*}{ Otras actividades } & Agricultura & 4 & 0 & 0 & 50 & \multirow[t]{4}{*}{0.37} \\
\hline & Industria & 0 & 0 & 0 & 0 & \\
\hline & Servicios & 54 & 60 & 100 & 0 & \\
\hline & Jubilado & 42 & 40 & 0 & 50 & \\
\hline \multirow[t]{3}{*}{ Tipo mano de obra } & Familiar & 65 & 59 & 84 & 25 & \multirow[t]{3}{*}{0.16} \\
\hline & Mixta & 23 & 23 & 8 & 63 & \\
\hline & Contratada & 12 & 18 & 8 & 12 & \\
\hline Usuario TIC & $\mathrm{Si} / \mathrm{No}^{2}$ & 57 & 41 & 17 & 63 & 0.06 \\
\hline \multirow[t]{3}{*}{ Evolución prevista rebaño } & Aumentar & 18 & 23 & 33 & 25 & \multirow[t]{3}{*}{0.38} \\
\hline & Mantener & 75 & 65 & 42 & 63 & \\
\hline & Decrecer & 7 & 12 & 25 & 12 & \\
\hline Presencia ovino & $\mathrm{Si} / \mathrm{No}^{2}$ & $17^{a}$ & $18^{a}$ & $92^{b}$ & $13^{a}$ & 0.00 \\
\hline Presencia caprino & $\mathrm{Sí} / \mathrm{No}^{2}$ & $1 \mathrm{a}$ & $0^{a}$ & $17^{b}$ & $0^{a}$ & 0.02 \\
\hline Presencia porcino reproductor & $\mathrm{Si} / \mathrm{No}^{2}$ & $45^{\mathrm{a}}$ & $100^{b}$ & $42^{a}$ & $25^{\mathrm{a}}$ & 0.00 \\
\hline Presencia porcino montanera & $\mathrm{Si} / \mathrm{No}^{2}$ & $39 a$ & $6^{a}$ & $33 a$ & $88^{b}$ & 0.00 \\
\hline Presencia equinos & $\mathrm{Si} / \mathrm{No}^{2}$ & 6 & 12 & 0 & 0 & 0.50 \\
\hline Monta dirigida ${ }^{3}$ & $\mathrm{Si} / \mathrm{No}^{2}$ & 28 & 46 & 20 & 38 & 0.60 \\
\hline Cubrición estacional & $\mathrm{Si} / \mathrm{No}^{2}$ & $29 a$ & $29 a$ & $0^{a}$ & $75^{b}$ & 0.01 \\
\hline Diagnóstico gestación & $\mathrm{Si} / \mathrm{No}^{2}$ & 13 & 12 & 0 & 38 & 0.11 \\
\hline Aporte concentrados antes destete & $\mathrm{Si} / \mathrm{No}^{2}$ & 55 & 71 & 50 & 50 & 0.62 \\
\hline Aporte concentrados después destete & $\mathrm{Si} / \mathrm{No}^{2}$ & 72 & 76 & 83 & 88 & 0.71 \\
\hline Oficialmente indemne tuberculosis 4 & $\mathrm{Si} / \mathrm{No}^{2}$ & 91 & 94 & 92 & 88 & 0.96 \\
\hline Oficialmente indemne brucelosis ${ }^{4}$ & $\mathrm{Si} / \mathrm{No}^{2}$ & 91 & 94 & 100 & 100 & 0.59 \\
\hline Plan vacunación vacas & $\mathrm{Si} / \mathrm{No}^{2}$ & 87 & 88 & 58 & 75 & 0.08 \\
\hline Plan vacunación terneros & $\mathrm{Si} / \mathrm{No}^{2}$ & $57^{b}$ & $29 a$ & $17^{a}$ & $25^{\mathrm{a}}$ & 0.01 \\
\hline Plan desparasitación vacas & $\mathrm{Si} / \mathrm{No}^{2}$ & 75 & 76 & 67 & 75 & 0.93 \\
\hline Plan desparasitación terneros & $\mathrm{Si} / \mathrm{No}^{2}$ & 51 & 65 & 33 & 50 & 0.43 \\
\hline
\end{tabular}

$a, b$ Letras distintas en la misma fila señalan diferencias significativas $(P<0.05)$.

${ }^{1}$ Ganaderos con más del $50 \%$ de las rentas procedentes de la actividad agraria.

${ }^{2}$ En las variables dicotómicas el porcentaje se refiere a las respuestas afirmativas.

${ }^{3}$ Control de la paternidad utilizando un único toro para cada lote de vacas.

${ }^{4}$ Calificación sanitaria oficial de acuerdo a la legislación española(17). 
El grupo 1 está constituido por 69 explotaciones (61\%) con una media de 122 ha y 40 hembras reproductoras. Se caracterizan por la coexistencia de varias especies ganaderas, destacando la asociación vacuno-porcino ibérico. La tasa de tierra en propiedad y la carga ganadera son altas.

El grupo 2 incluye 17 explotaciones ( $15 \%$ ) con una media de 255 ha y 64 vacas. El ganado principal de estas explotaciones es el porcino reproductor, dedicado a la producción de lechones para el cebo intensivo o la montanera en otras explotaciones. Las explotaciones de este grupo se sitúan mayoritariamente en la zona noroeste del área de estudio, con los suelos más pobres y la densidad de arbolado más baja. Por ello, la carga ganadera excluyendo las cerdas reproductoras es la menor de todos los grupos y, en aquellas explotaciones en las que hay cerdos en montanera, el número de cerdos/ha también es menor que en el resto de grupos.

El grupo 3 está compuesto de 12 explotaciones (11\%) con una media de 174 ha y 31 vacas. La ganadería principal en estas explotaciones es el ovino. El cerdo ibérico también está presente, siendo la media del número de cerdas reproductoras muy inferior a la media del número de cerdos en montanera, debido a que en estas explotaciones las reproductoras se usan fundamentalmente para producir los lechones que se cebarán en montanera en la propia explotación. La productividad del vacuno en términos de terneros destetados por vaca y año es la más baja de todos los grupos, y las prácticas de manejo son las menos tecnificadas: cubrición anual y escaso uso de los planes de vacunación. La tasa de tierras arrendadas es la más alta de todos los grupos.

El grupo 4 lo componen 8 explotaciones $(7 \%)$ con una media de 425 ha y 124 vacas. El vacuno es la actividad más importante en este caso, si bien el cerdo ibérico de montanera está presente en la mayoría de las explotaciones, siendo el número de cerdos/ha bajo. La carga ganadera total es la más baja de todos los grupos. El manejo reproductivo del vacuno es intensivo, siendo habitual el uso de la monta dirigida, la cubrición estacional e, incluso, el diagnóstico de gestación. El porcentaje de mano de obra familiar es el más bajo.

El análisis de conglomerados ha identificado 8 explotaciones (7\%) que no forman parte de los grupos definidos anteriores, constituyendo cada una de ellas un grupo diferente. Se trata de cuatro explotaciones de más de 1,000 ha, dos explotaciones de entre 500 y 1,000 ha, y dos explotaciones con menos de $100 \mathrm{ha}$; todas ellas con diferentes tipos de ganado, de manejo y de régimen de propiedad. 


\section{" Discusión N}

Se observó una gran diversidad en las características y los sistemas de producción de las explotaciones estudiadas. La aproximación censal y micro usada en este trabajo, encuestando casi todas las explotaciones existentes en un área pequeña, no es habitual en los estudios de caracterización y tipificación de explotaciones ganaderas ${ }^{(18)}$, pero ha resultado adecuada para registrar la variabilidad existente ${ }^{(19,20)}$, permitiendo identificar incluso las tipologías de explotaciones menos habituales en el área de estudio, cuya probabilidad de ser incluidas en un muestreo estratificado es baja. No obstante, si bien existen explotaciones muy diversas, en la zona destaca cuantitativamente la tipología, identificada como grupo 1. Los trabajos basados en muestreos suelen encontrar tipologías de explotaciones con un tamaño similar, ya que las explotaciones se seleccionan con el objetivo de maximizar la variabilidad. En áreas pequeñas esto puede distorsionar la percepción de la importancia relativa de las diferentes tipologías.

Varios estudios han concluido que el tamaño es uno de los factores que mejor explican la variabilidad entre explotaciones ${ }^{(6,8)}$. El tamaño medio de las explotaciones encuestadas es mucho menor que el descrito en estudios previos para el suroeste de España ${ }^{(6)}$, para Extremadura $^{(21,22)}$ y para Andalucía ${ }^{(7)}$. En el área de estudio, las características de los sistemas de propiedad y herencia de la tierra determinan un tamaño de las explotaciones de dehesa menor que en otras zonas de España y Portugal, donde la superficie promedio es de 500 a 600 ha. No obstante, Castillo ${ }^{(23)}$ registró, con datos de 2002, una superficie promedio de las explotaciones de dehesa de Los Pedroches asociadas al cebadero cooperativo que supera en 100 ha a la superficie promedio observada en este estudio, siendo además el número de vacas aproximadamente el doble. Este dato, junto al bajo porcentaje de las explotaciones encuestadas que superan las 300 ha (grupo 4), parece indicar que las explotaciones de mayor tamaño y carácter empresarial de la zona, o bien no se han asociado, o bien han dejado de participar en el sistema cooperativo. Este tipo de explotaciones tienen más facilidad para seguir una estrategia de venta o cebo de los terneros a título individual, tanto desde el punto de vista técnico como financiero, siendo las ventajas de la integración cooperativa menos evidentes para estos ganaderos.

La diversificación de las especies ganaderas explotadas es otro factor de variabilidad entre explotaciones. La importancia relativa de las distintas especies puede tener un efecto importante en la sostenibilidad de los sistemas productivos, tanto desde el punto de vista económico como medioambiental ${ }^{(24)}$. El peso del vacuno en las explotaciones encuestadas, pese a disponer de un sistema cooperativo de cebo, sacrificio y comercialización, es menor que el descrito por otros autores ${ }^{(8,21)}$, debido a la importancia del porcino. El cerdo ibérico es 
el ganado más rentable de la dehesa por los altos precios de mercado de sus productos ${ }^{(25)}$. La abundancia de dehesas de encinas e industrias cárnicas en las comarcas estudiadas favorece que el cerdo ibérico de montanera esté presente en el $40 \%$ de las explotaciones. Además, cuando las características físicas de las fincas no son adecuadas para la montanera debido a la baja densidad de arbolado (grupo 2), la estrategia de los ganaderos para mejorar la rentabilidad suele ser producir lechones, en instalaciones intensivas o pequeños cercados, que puedan ser engordados como cerdos de montanera en otras explotaciones o, si las condiciones de mercado son favorables, que puedan cebarse en base a pienso compuesto en su propia explotación. La tercera especie ganadera de importancia en la zona es el ovino. La oveja es el animal que mejor aprovecha los pastos de la dehesa, pero el coste de la mano de obra asociada a su manejo ha llevado a su sustitución paulatina por el vacuno, que está más adaptado al pastoreo libre y es menos susceptible al ataque de depredadores ${ }^{(26,27)}$. No obstante, en algunas explotaciones (grupo 3) el ovino aún es la especie ganadera más importante. La presencia del ovino en las dehesas suele asociarse en la literatura científica a la existencia de pagos acoplados de la Política Agraria Común ${ }^{(4)}$. No obstante, en este estudio se observa la influencia de otros factores, relativos a las características socioeconómicas y culturales de las explotaciones, cuya importancia se ha destacado en estudios recientes ${ }^{(28)}$.

Se observa una correlación con el sistema de tenencia de la tierra, teniendo las explotaciones del grupo 3 un porcentaje de superficie arrendada muy superior al resto, mayor al $70 \%$. La incertidumbre sobre la continuidad de la actividad ganadera en una finca arrendada es mayor que en una finca en propiedad, siendo los rebaños de ovino más flexibles que los de vacuno, tanto en lo que se refiere a su traslado entre fincas como a su capacidad de crecer o menguar en número de cabezas. Otros estudios también han registrado esta correlación entre ovino y arrendamiento, si bien no la han discutido explícitamente ${ }^{(21)}$. Por otro lado, el perfil de los ganaderos de ovino puede definirse como "tradicional"; el promedio de usuarios de las tecnologías de la información y la comunicación (TIC) es el menor de todos los grupos, mientras que el porcentaje de agricultores a título principal y el porcentaje de mano de obra familiar son los más altos, si bien las diferencias entre grupos no son estadísticamente significativas.

El nivel de intensificación de la producción también es un factor de variabilidad entre explotaciones, tanto en lo referente a la carga ganadera como al manejo. Los valores de carga ganadera observados son, en general, muy superiores a los tradicionales en los sistemas de dehesa $^{(2)}$ y a los reportados por otros autores ${ }^{(6,7,27)}$. Las mayores cargas corresponden a las explotaciones del grupo 2 debido a la existencia de cerdas reproductoras. En cuanto a la carga sobre el sistema pastoral, no existen diferencias significativas entre tipologías, pero se observa una correlación negativa con la superficie total de la explotación. La superficie reducida es uno de los factores de intensificación de las explotaciones encuestadas. La estrategia de los ganaderos cuando el terreno es limitado suele ser aumentar el tamaño de los rebaños, aún a costa de una mayor dependencia de recursos externos ${ }^{(29)}$. 
El manejo de la alimentación es más intensivo que el descrito en estudios previos, destacando el uso agrícola de las dehesas. Las explotaciones cultivan anualmente casi el $50 \%$ de la superficie cultivable de la que disponen, estando este valor en torno al $10 \%$ en otros estudios $^{(6,22)}$. No se observan diferencias entre grupos en el porcentaje de tierra cultivada anualmente, pero existe una correlación con el peso relativo del vacuno, ya que las vacas requieren de más alimentación suplementaria en épocas de escasez de pastos que las ovejas, sobre todo cuando no son de razas autóctonas. La alimentación de los terneros también se ha intensificado, ya que la mayoría de los ganaderos les aportan concentrados antes y, sobre todo, después del destete. Los ganaderos son conscientes de que acostumbrar a los terneros al consumo de pienso antes de su entrada en el cebadero cooperativo tiene un efecto positivo en la fase de cebo y, por ende, en su beneficio económico.

En cuanto a la reproducción, un factor importante son las razas explotadas. El porcentaje de explotaciones con vacas autóctonas es menor que el observado por otros autores ${ }^{(6,21,22)}$. Aunque dichos estudios citan la importancia creciente del ganado Charolais y Limousin, en ningún caso reportan una presencia tan abundante como la observada en el área de estudio. Muchos ganaderos consideran que las vacas autóctonas son superiores a las de razas especializadas en las condiciones de la dehesa en cuanto a aprovechamiento de los recursos, intervalo entre partos y peso de los terneros al destete, momento en que se venden los terneros en los sistemas tradicionales. Sin embargo, la participación en el cebadero cooperativo supone avanzar en la cadena de valor, y ello favorece la elección de razas especializadas, o sus cruces con las autóctonas, debido a su mayor velocidad de crecimiento y conformación de la canal ${ }^{(30)}$, aspectos de gran importancia en las fases de cebo y sacrificio, y que suponen un mayor pago por cada ternero individual. Los cambios de genética se suelen realizar mediante cruces por absorción con toros de razas especializadas, lo que da lugar a la coexistencia de animales con diferentes grados de cruzamiento tanto en las explotaciones como en el cebadero ${ }^{(30)}$.

En lo que se refiere al uso de técnicas de gestión reproductiva, el manejo puede considerarse extensivo, siendo la cubrición estacional y el diagnóstico de gestación menos habituales que en otros estudios ${ }^{(6)}$. En este caso se observan diferencias significativas entre tipologías de explotaciones. En las explotaciones del grupo 4, que tienen los rebaños de vacuno más grandes, el uso de ambas técnicas es más habitual. En estas explotaciones, los partos de verano suponen mayores necesidades de mano de obra, fundamentalmente contratada, y de alimentación suplementaria, por lo que los ganaderos suelen optar por evitar las montas durante algunos meses al año, aún a costa de aumentar las posibilidades de que algunas vacas queden vacías. Por el contrario, los ganaderos del resto de grupos, que tienen rebaños de vacas de menor tamaño y más mano de obra familiar, prefieren minimizar el número de vacas vacías haciendo uso de la cubrición continua. Cabe señalar que la cubrición estacional acentúa la estacionalidad de las entradas de terneros en el cebadero cooperativo (Figura 1), lo cual puede suponer problemas logísticos y de comercialización. 
Figura 1: Número terneros entregados a cebadero por mes

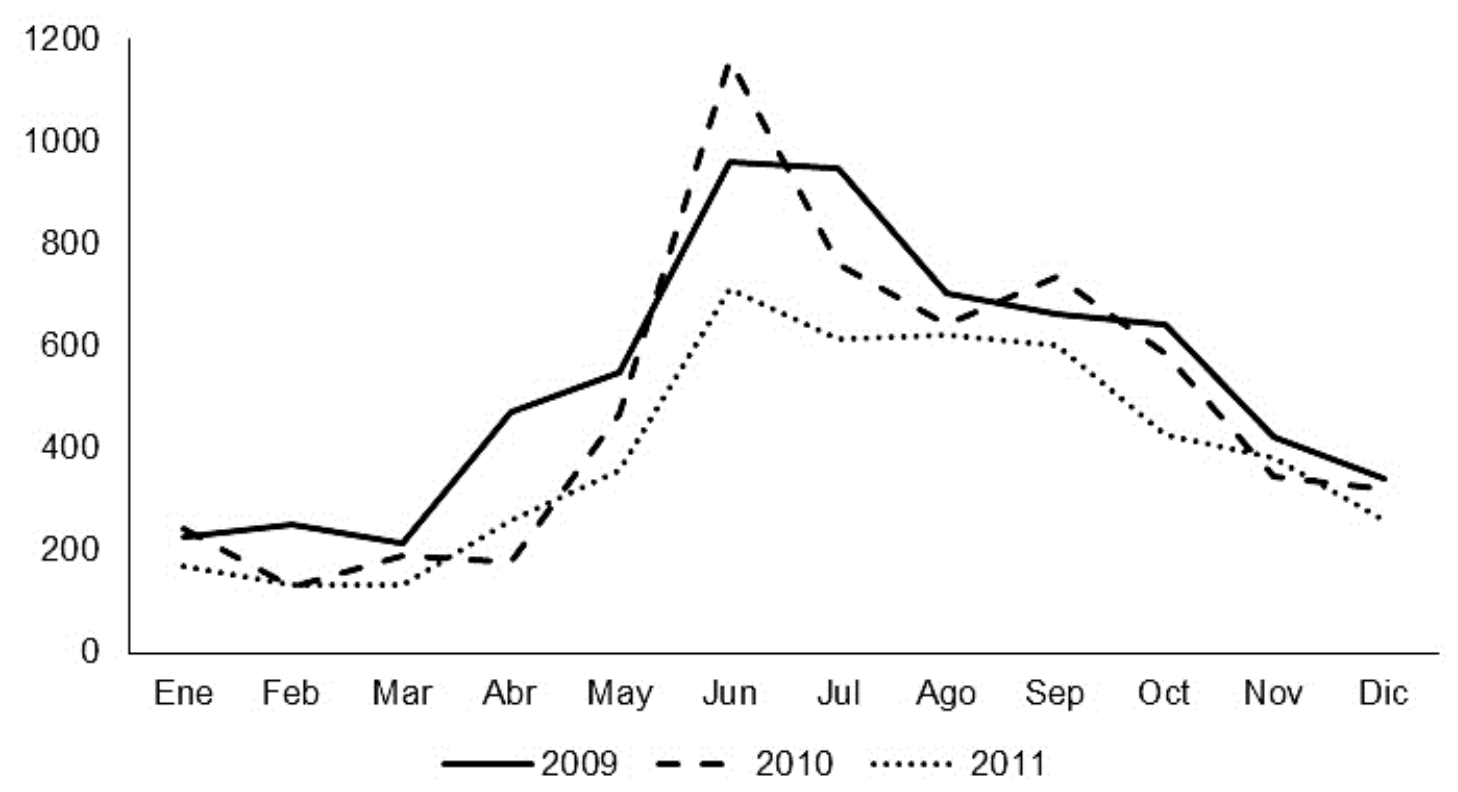

En lo referente al manejo sanitario, la situación de las explotaciones encuestadas es, en general, buena. Solamente se observan diferencias significativas entre grupos en la vacunación de los terneros. Esta práctica es más habitual en las explotaciones del grupo 1, donde el vacuno es la producción más importante. En las del grupo 4, si bien el vacuno también es la especie más importante, la vacunación de los terneros es poco frecuente, posiblemente debido a que, dado que el número de terneros producidos es grande, la vacunación es un coste importante y el impacto de las posibles bajas es menor.

\section{"Conclusiones e implicaciones \|}

En el caso estudiado, las explotaciones asociadas a la cooperativa de cebo, sacrificio y comercialización de terneros presentan algunas características poco habituales en los sistemas de dehesa, destacando el pequeño tamaño y la importancia de la mano de obra familiar. Son este tipo de explotaciones las que presentan una mayor dependencia de las cooperativas para avanzar en la cadena de valor, ante las dificultades que les supone afrontar 
el cebo y la comercialización de forma individual. Pese a su proximidad geográfica y su integración en un modelo de comercialización común, las explotaciones presentan sistemas de producción diversos. La superficie disponible, el sistema de propiedad y el potencial productivo de las fincas (precipitación y calidad del suelo) determinan la variabilidad entre explotaciones, destacando las diferencias en las estrategias de diversificación de la producción y en los sistemas de manejo. Las explotaciones presentan, en general, un nivel de intensificación elevado; el aumento de la carga ganadera y la sustitución de razas autóctonas por animales especializados con mayores necesidades de suplementación son factores de intensificación destacados.

\section{N Agradecimientos}

Este trabajo ha sido financiado por el Ministerio de Agricultura, Pesca, Alimentación y Medio Ambiente (20130020000783) y la Comisión Europea (LIFE 11/BIO/ES/000726). Los autores agradecen los comentarios de los revisores anónimos para la mejora del manuscrito original.

\section{- Literatura citada:}

1. Gastó J, Calzado C, Carbonero MD, de Pedro E, Fernández P, Garrido A, Gómez A, et al. Sostenibilidad de las dehesas: documento de reflexión. Dos Torres, España: GDR Los Pedroches; 2010.

2. Plieninger T, Wilbrand C. Land use, biodiversity conservation, and rural development on the dehesas of Cuatro Lugares, Spain. Agrofor Syst 2001;51:23-34.

3. Ferraz MI, Azeda C, Pinto T. Management of montados and dehesas for High Nature Value: an interdisciplinary pathway. Agrofor Syst 2016;90(1):1-6.

4. Bernués A, Ruiz R, Olaizola A, Villalba D, Casasús I. Sustainability of pasture-based livestock farming in the European Mediterranean context: synergies and trades-off. Livest Sci 2011;139:44-57. 
5. Kristjanson P, Krishna A, Radeny M, Kuan J, Quilca G, Sánchez A, León C. Poverty dynamics and the role of livestock in the Peruvian Andes. Agric Syst 2007;94:294-308.

6. Milán MJ, Bartolomé J, Quintanilla R, García MD, Espejo M, Herráiz PL, Sánchez JM, Piedrafita J. Structural characterization and typology of beef cattle farms of Spanish wooded rangelands (dehesas). Livest Sci 2006;99:197-209.

7. Perea J, García A, Acero R, Valerio D, Rodríguez V. Caracterización productiva del vacuno ecológico en Andalucía. Arch Zootec 2007;56 (1):517-521.

8. García A, Perea J, Acero R, Angón E, Toro P, Rodríguez V, Gómez G. Caracterización estructural de los sistemas ganaderos de las dehesas andaluzas. Arch Zootec 2010;59:577-588.

9. Escribano AJ, Gaspar P, Mesías FJ, Escribano M. The role of level of intensification, productive orientation and self-reliance in extensive beef cattle farms. Livest Sci 2016;193:8-19.

10. Gaspar P, Escribano M, Pulido F, Rodríguez A, Mesías FJ, Pulido AF. El papel de la gestión cooperativa en la mejora del funcionamiento técnico-económico de las explotaciones ovinas. Arch Zootec 2016;65(251):333-339.

11. Theuvsen L, Franz A. The role and success factors of livestock trading cooperatives: lessons from German pork production. IAMA 2007;10(3):90-112.

12. Horrillo A, Escribano M, Mesías FJ, Elghannam A, Gaspar P. Is there a future for organic production in high ecological value ecosystems? Agrofor Syst 2016;143:114125.

13. Martín M, Espejo M, Plaza J, López T. Cálculo de la carga ganadera en la dehesa. In: Martín M, Campos P editors. Conservación y desarrollo de las dehesas portuguesa y española. Ministerio de Agricultura, Pesca y Alimentación, España: 1987:239-257.

14. Escribano M, Pulido F, Rodríguez A, Mesías FJ. Determination of energetic resources that cover cattle food needs in dehesa systems. Arch Zootec 1996;45:379-393.

15. Solano C, Bernués A, Rojas F, Joaquín N, Fernández W, Herrero M. Relationships between management intensity and structural and social variables in dairy and dualpurpose systems in Santa Cruz. Agric Syst 2000;65:159-177.

16. DOUE. Council Regulation (EC) No. 73/2009 of 19 January 2009 establishing common rules for direct support schemes for farmers under the Common Agricultural Policy and establishing certain support schemes for farmers. 
17. BOE. Real Decreto 2611/1996, de 20 de diciembre, por el que se regulan los programas nacionales de erradicación de enfermedades de los animales.

18. Madry W, Mena Y, Roszkowska B, Gozdowski D, Hryniewski R, Castel JM. An overview of farming system typology methodologies and its use in the study of pasturebased farming systems: a review. Span J Agric Res 2013;1 (2):316-326.

19. Carmona A, Nahuelhual L, Echevarría C, Báez A. Linking farming systems to landscape change: an empirical and spatially explicit study in southern Chile. Agric Ecosyst Environ 2010;139:40-50.

20. Castel JM, Madry W, Gozdowski D, Roszkowska B, Dabrowski M, Lupa W, Mena Y. Family dairy farms in the Podlaise province, Poland: farm typology according to farming system. Span J Agric Res 2010;8:946-961.

21. Gaspar P, Mesías FJ, Escribano M, Rodríguez A, Pulido F. Economic and management characterization of dehesa farms: implications for their sustainability. Agrofor Syst 2007;71:151-162.

22. Horrillo A, Mesías FJ, Gaspar P, Escribano M. La explotación de vacuno ecológico en sistemas de dehesa del SO de España: análisis de parámetros técnicos. Arch Zootec 2015;64: 307-310.

23. Castillo M. Eficiencia técnica de la producción de vacuno de carne en la dehesa. REEAP 2006;212:139-154.

24. Rook AJ, Dumont B, Isselstein J, Osoro K, Wallis MF, Parente G, Mills J. Matching type of livestock to desired biodiversity outcomes in pastures, a review. Biol Conserv 2004;119(2):137-150.

25. Gaspar P, Mesías FJ, Escribano M, Pulido F. Sustainability in Spanish extensive farms (dehesas): an economic and management indicator-based evaluation. Rangeland Ecol Manag 2009;62:153-162.

26. Ortuño SF, Fernández JL. Perspectivas económicas de las producciones ganaderas extensivas en las áreas desfavorecidas ante la liberalización de los mercados. Rev Esp Econ Agr 1995;174:165-188.

27. Escribano M, Rodríguez A, Mesías FJ, Pulido F. Niveles de cargas ganaderas en la dehesa extremeña. Arch Zootec 2002;51:315-326.

28. Torralba M, Oteros E, Moreno G, Plieninger T. Exploring the role of management in the coproduction of ecosystem services from Spanish wooded rangelands. Rangeland Ecol Manag 2017 [in press]. 
29. Plieninger T, Modolell J, Konold W. Land manager attitudes toward management, regeneration and conservation of Spanish holm oak savannas (dehesas). Landscape Urban Plan 2004;66:185-198.

30. Maroto F, Santos R, Gómez J, Gómez A. Caracterización de la variabilidad intrarracial de los parámetros productivos de añojos cebados en el sur de España. Panea B, et al editores. XVI Jornadas AIDA Producción Animal; 2015:39-41. 